Revista do Departamento de Geografia
Universidade de São Paulo
www.revistas.usp.br/rdg

\title{
Qualidade Ambiental e Promoção de Saúde: Um Estudo Sobre o Parque do Povo de Presidente Prudente - SP
}

\author{
Environmental Quality and Health Promotion: \\ A Study on The Parque do Povo in Presidente Prudente - SP
}

\author{
Alba Regina Azevedo Arana \\ Universidade do Oeste Paulista \\ alba@unoeste.br \\ Fernanda Berguerand Xavier \\ Universidade do Oeste Paulista \\ fernandaberguerand@hotmail.com
}

Resumo: Evidências encontradas em estudos recentes indicam que a oferta de parques urbanos está associada a benefícios na saúde da população local. Um mecanismo proposto para essa questão é que os parques influenciam positivamente a auto percepção de saúde do indivíduo e incentiva a prática de atividades físicas. Em Presidente Prudente - SP, o Parque do Povo é o principal espaço verde público de lazer e esportes da cidade. Qualidade ambiental e espaços verdes urbanos estão intimamente relacionados, e entre inúmeros benefícios, esses locais promovem oportunidades para a prática de atividades físicas (AF). Dessa forma, este artigo tem como objetivo verificar a relação entre a qualidade ambiental urbana e a prática de $\mathrm{AF}$, e discutir como o ambiente pode agir em estratégias ambientais de promoção de saúde. A metodologia consistiu em uma abordagem qualiquantitativa e a técnica foi o uso de questionário aplicado a 200 frequentadores do parque maiores de 18 anos. A coleta de dados foi realizada durante o primeiro semestre de 2015. Os questionários mostraram que a maioria da população residia nas proximidades do parque, e os motivos relacionados a saúde foram os mais destacados para a prática de AF. O fator "ambiente verde" foi o mais valorizado no quesito "motivação" para escolha do local. Concluiu-se que os parques urbanos trazem benefícios à saúde, mas não garantem por si sós o aumento do nível de atividade física.

Palavras-chave: Parques Urbanos; Atividade Física; Qualidade Ambiental; Saúde.

\begin{abstract}
Evidence from recent studies indicate that the supply of urban parks is associated with health benefits the local population. A proposed mechanism for this issue is that the parks positively influence the self-perceived health of the individual and encourages the practice of physical activities. The People's Park of Presidente Prudente is the main public green space for recreation and sports of the city. Environmental quality and urban green spaces are closely related, and among many benefits, these sites promote opportunities for physical activities (PA). Thus, this article aims to investigate the relationship between urban environmental quality and $P A$ practice, and discuss how the environment can act on environmental strategies for health promotion. The methodology consisted of a qualitative-quantitative approach and the technique was the use of a questionnaire applied to 200 frequent attendees park with age over 18 years. Data collection was carried out during the first halve of 2015. The questionnaires showed that most of the population lived near the premises, and health-related reasons were the most outstanding for PA practice. The "green environment" factor was the most valued in the item "motivation" to choose the location. It was concluded that urban parks bring health benefits, but do not guarantee on their own an increase of physical activity level.
\end{abstract}

Keywords: Urban Parks; Physical Activity; Environmental Quality; Health. 


\section{INTRODUÇÃO}

A inatividade física possui uma relação causal com quase $30 \%$ de todas as mortes por cardiopatia, câncer de cólon e diabetes (MCARDLE, 2011). Em qualquer idade, tornar-se fisicamente mais ativo retarda a mortalidade relacionada a essas e outras causas. As pessoas com estilo de vida mais saudáveis sobrevivem por mais tempo, e o risco de incapacitação e de procura por assistência médica é protelado (MCARDLE, 2011).

O sedentarismo é apontado como problema de saúde púbica no Brasil. Dados do Instituto Brasileiro de Geografia Estatística (IBGE) em pesquisa divulgada em Dezembro de 2014 aponta que 46\% da população com idade superior a 18 anos é sedentária, não atingindo a recomendação de 150 minutos de atividade física por semana, seja nos momentos de lazer, no trabalho ou nos deslocamentos até o trabalho (PNS, 2014). Ainda segundo a pesquisa, o percentual de pessoas que praticam atividades físicas no lazer diminui de acordo com a faixa etária. De 18 a 24 anos, a proporção é de 35,3\%. Essas taxas vão caindo para 25,5\% (de 25 a 39 anos), $18,3 \%$ (de 40 a 59 anos) e 13,6\% (de 60 anos ou mais).

Intervenções de saúde direcionadas à mudança comportamental do indivíduo para torna-lo fisicamente ativo têm alcançado mudanças positivas, mas os efeitos têm se mostrado pequenos e de curto prazo, não havendo avanço substancial nos níveis de atividade física da população (BEDIMO-RUNG et al., 2005). Ademais, pesquisadores têm constatado que a vida em ambientes mais naturais influencia positivamente a auto percepção de saúde das pessoas e leva a um menor risco de mortalidade (MAAS et al., 2008). De acordo com Branas et al. (2011), uma estratégia multinível que combine indivíduo e meio ambiente pode atingir metas maiores e mais sustentáveis, pois influenciam maior número de pessoas por um período de tempo significativamente superior aos programas individuais de saúde.

As grandes concentrações urbanas, a redução dos espaços livres, assim como a revolução tecnológica criaram cenários que predispõem ao estilo de vida inativo (COLLET et al., 2008). Ainda que constitua apenas um único fator, parques urbanos são espaços públicos comuns que podem desempenhar um papel significativo no aumento dos níveis de atividade física da população. Eles fornecem locais propícios para que as pessoas caminhem ou corram, muitas vezes possuem instalações específicas para esportes, exercícios e outras atividades vigorosas, oferecendo a oportunidade de incluir atividades físicas nos momentos de lazer de forma gratuita.

Fredric Olmstead (1999, apud COHEN et al., 2007), o "pai dos parques urbanos" observa que esses devem ser construídos como locais onde os moradores da cidade possam experimentar a beleza da natureza, respirar ar fresco e ter um lugar receptivo para ouvir música e apreciar a arte, praticar atividades esportivas, bem como conviver com amigos e vizinhos. Sendo assim, parques urbanos podem desempenhar um papel na facilitação da atividade física, mas não necessariamente fazê-lo, por também proporcionar oportunidades para que as pessoas se envolvam em comportamento sedentário (WALKER, 2004; apud COHEN et al., 2007).

A escassez de informações quanto à forma de utilização e os fatores que podem facilitar ou impedir a utilização dos parques de maneira ativa, traz à tona a necessidade de compreensão dos fatores que possam intervir na ocupação de parques urbanos enquanto espaço de lazer (COLETT et al., 2008). Desta forma, este artigo tem como objetivo apresentar a importância dos parques urbanos para a promoção de saúde e práticas de atividades físicas no espaço urbano. E ainda apresentar a importância do Parque do Povo em Presidente Prudente para a realização de $\mathrm{AF}$, suas formas de utilização e sua relevância com a qualidade urbana ambiental. Este trabalho parte da seguinte reflexão: em que extensão os parques compreendem uma chave para a redução do sedentarismo? Quais as características determinantes desses locais que atuam como promotoras de saúde através da prática de atividades físicas?

\section{METODOLOGIA}

A metodologia utilizada para a realização deste trabalho está fundamentada na pesquisa bibliográfica e trabalho de campo. O trabalho de campo objetivou obter Informações sobre quem são os potenciais frequentadores dos parques públicos, o que eles buscam e quais as atividades que realizam nesses ambientes podem elucidar a real contribuição desses locais na estratégia de promoção de saúde bem como potencializar seus benefícios. No entanto, a diversidade de resultados e variáveis encontradas nesses estudos têm produzido resultados mistos (ORD et al., 2013), não concebendo padrões consistentes de associação. 
Utilizou-se o método de amostragem probabilístico, através da aplicação aleatória de questionário semiaberto estruturado especificamente para atender os objetivos propostos pela pesquisa (questionário cadastrado e aprovado no comitê de ética - CAE 36161414.0.0000.5515). Uma amostra é probabilística quando qualquer membro de uma população alvo tem uma probabilidade conhecida $(>0)$ de ser incluído na amostra. O objetivo de uma amostra probabilística é eliminar a subjetividade e obter uma amostra que seja imparcial e representativa da população alvo (AAKER, 2001).

Os questionários foram aplicados aos frequentadores do local, maiores de 18 anos e que estavam praticando alguma $\mathrm{AF}$, em horários e dias da semana aleatórios durante os meses de abril a setembro de 2015. O sigilo e o direito de recusa foram garantidos durante toda essa etapa.

As questões levantadas abordaram: (a) Características dos usuários do parque (BROOMHALL, 1996); (b) Motivação para a prática do exercício físico (MARKLAND e INGLEDEW, 1997; MOUTÃO, 2005); (c) Motivos que trazem o indivíduo até o parque para a realização dessa prática (TINSLEY et al., 2002; GILES-CORTI et al., 2005); (d) Fatores psicológicos que influenciam a preferência pelo local (GILES-CORTI et al., 2005)

Todas as análises estatísticas foram feitas através da utilização de programação específica em linguagem Python e SQL (Structured Query Language). Os dados foram verificados duas vezes pelo investigador principal para a verificação e validação dos dados. A verificação de dados também incluiu uma avaliação dos meios, desvios-padrão, frequências e intervalos interquartis para ajudar a identificar possíveis erros na entrada de dados. Os objetivos do estudo foram abordados usando vários modelos de regressão linear.

As notas de campo, com base em observações sistemáticas e aplicação de 200 questionários foram analisadas tematicamente com relação às variáveis e objetivos do estudo. As anotações foram então classificadas, e os temas e descobertas observacionais foram posteriormente discutidos para minimizar viés de pesquisador na interpretação dos resultados.

\section{ATIVIDADE FÍSICA, SAÚDE E QUALIDADE DE VIDA}

É importante analisarmos, do ponto de vista da saúde, o potencial que a prática regular de atividades físicas oferece na estratégia de promoção de saúde e qualidade de vida. Para isso, faremos a conceituação de alguns termos utilizados durante este estudo para maior esclarecimento do tema, e também apresentaremos os benefícios comprovados pela ciência que a atividade física estruturada proporciona aos indivíduos que dela se utilizam.

A atividade física é tradicionalmente definida como qualquer movimento corporal produzido pela contração dos músculos esqueléticos que resulte em um aumento substancial em relação ao dispêndio de energia em repouso (ACSM, 2010). Com frequência, os termos atividade física e exercício físico são erroneamente utilizados como sinônimos. A American College of Sports Medicine (ACSM) ressalta essa diferença, particularizando o exercício físico como um tipo de atividade física que consiste em movimentos corporais planejados, estruturados e repetitivos realizados para aprimorar ou preservar um ou mais componentes da aptidão física.

A definição apresentada pelo Manifesto do Cirurgião Geral dos Estados Unidos em 1996 e citada por Araújo e Araújo (2000) considera como atividade física todo movimento corporal com gasto energético acima dos níveis de repouso, incluindo atividades decorrentes do dia-a-dia, como tomar banho, vestir-se; atividades realizadas durante o trabalho, como andar, carregar, levantar-se; e também as atividades de lazer, como a prática de exercícios, esportes, danças, etc. Shephard (1990) define atividade física como qualquer movimento corporal produzido pelos músculos esqueléticos que resultem em gasto energético, não se preocupando com a magnitude desse gasto de energia. Esses autores também diferenciam atividade física e exercício físico a partir da intencionalidade do movimento, considerando que o exercício físico é um subgrupo das atividades físicas, que é estruturado, planejado e repetitivo, objetivando a manutenção ou otimização da aptidão física.

A ACSM define aptidão física como um conjunto de atributos ou características que as pessoas possuem ou adquirem e se relacionam com a capacidade de realizar uma atividade física. Esses atributos são subdivididos em "Componentes de Aptidão Física Relacionados à Saúde" e "Componentes da Aptidão Física Relacionados às Habilidades" (Quadro 1): 
Quadro 1: Componentes de Aptidão Física Relacionados à Saúde e Relacionados às Habilidades.

Componentes de Aptidão Física Relacionados à Saúde e Relacionados às Habilidades

\section{COMPONENTES DE APTIDÃO FÍSICA RELACIONADOS À SAÚDE}

- Endurance cardiovascular: Capacidade dos sistemas circulatório e respiratório de fornecerem oxigênio durante uma atividade física sustentada.

- Composição corporal: As quantidades relativas de músculo, gordura, osso e outras partes vitais do organismo.

- Vigor muscular: A capacidade do músculo de exercer força.

- Endurance muscular: A capacidade do músculo de continuar se contraindo sem contrair fadiga.

- Flexibilidade: A amplitude de movimento disponível em uma articulação.

\section{COMPONENTES DA APTIDÃO FÍSICA RELACIONADOS ÀS HABILIDADES}

- Agilidade: A capacidade de modificar a posição do corpo no espaço com velocidade e acurácia.

- Coordenação: A capacidade de utilizar os sentidos, como a vista e a audição, juntamente com outras partes corporais para realizar as tarefas com regularidade e exatidão.

- Balanço (equilíbrio): A manutenção do equilíbrio na condição estacionária ou em movimento.

- Potência: A capacidade ou o ritmo com que se consegue realizar um trabalho.

- Tempo de reação: $\mathrm{O}$ tempo transcorrido entre a estimulação e o início da reação a essa estimulação.

- Velocidade: A capacidade de realizar um movimento dentro de um curto período.

Fonte: Adaptado de ACSM's 2010, p. 2.

Em complemento a essas definições, a questão da aptidão física é abordada por Guedes (1996) em seu capítulo nas "Orientações Básicas sobre Atividades Físicas e Saúde para Profissionais das Áreas de Educação e Saúde", definindo-a como "um estado dinâmico de energia e vitalidade que permite a cada um não apenas a realização das tarefas do cotidiano, as ocupações ativas das horas de lazer e enfrentar emergências imprevistas sem fadiga excessiva, mas, também, evitar o aparecimento das funções hipocinéticas, enquanto funcionando no pico da capacidade intelectual e sentindo uma alegria de viver". Propõe também que a aptidão física seria a capacidade de realizar esforços físicos sem fadiga excessiva, garantindo a sobrevivência de pessoas em boas condições orgânicas no meio ambiente em que vivem.

Dentro desse contexto, a epidemiologia da atividade física é abordada por McArdle (2011) em seu capítulo "Atividade Física, Saúde e Envelhecimento", e definições são aplicadas para caracterizar os padrões de comportamento e suas consequências nos grupos investigados. Para o autor, a terminologia relevante inclui o seguinte: (a) Atividade física: Movimento corporal produzido pela contração muscular e que faz aumentar o dispêndio de energia; (b) Exercício: Atividade física planejada, estruturada, repetitiva e intencional; (c) Aptidão física: Atributos relacionados com a maneira pela qual se executa uma atividade física; (d) Saúde: Bem estar físico, mental e social, e não apenas ausência de doença.

A definição de saúde concentra-se no amplo espectro de bem-estar e varia da ausência completa de saúde (quase morte) aos mais altos níveis de função fisiológica. Com frequência, essas definições desafiam nossa maneira de medir e quantificar objetivamente a saúde e a atividade física. Entretanto, elas proporcionam uma ampla perspectiva para estudar o papel da atividade física na saúde e na doença.

Para McArdle (2011), a tendência na avaliação da aptidão física durante os últimos 40 anos deixa de enfatizar os testes que realçam o desempenho motor e a aptidão atlética (i.e., velocidade, potência, equilíbrio e agilidade) e passa a focar as capacidades funcionais, relacionadas com a boa saúde global e com a prevenção de doenças. $\mathrm{O}$ autor define quatro componentes mais comuns da atividade física relacionada com a saúde, sendo estes: aptidão aeróbica e/ou cardiovascular, a composição corporal, a endurance dos músculos abdominais e a flexibilidade da região lombossacra e da musculatura posterior da coxa (hamstring). 
Após definirmos atividade física, exercício e aptidão física, esclarecemos mais uma vez que a saúde não se caracteriza apenas como um estado de ausência de doenças, mas como um estado geral de equilíbrio no indivíduo, nos diferentes aspectos e sistemas que caracterizam o homem: biológico, psicológico, social, emocional, mental e intelectual, resultando em sensação de bem-estar.

\section{QUALIDADE URBANA AMBIENTAL COMO FATOR DETERMINANTE DE SAÚDE}

A qualidade ambiental urbana é um conceito de difícil definição, e está atrelada a vários fatores de infraestrutura, desenvolvimento econômico-social e aqueles ligados à questão ambiental das cidades. Oliveira (1983, apud GOMES; SOARES, 2004) salienta que a qualidade de vida está intimamente ligada à qualidade ambiental, pois vida e meio ambiente são inseparáveis, o que não significa que o meio ambiente determina as várias formas e atividades de vida ou que a vida determina o meio ambiente. Na verdade, o que existe é uma interação entre ambos que variam de escala em tempo e lugar.

A partir do início da década de 1990, parece consolidar-se um consenso entre os estudiosos quanto a dois aspectos relevantes do conceito de qualidade de vida: subjetividade e multidimensionalidade. A subjetividade trata-se de considerar a percepção da pessoa sobre o seu estado de saúde e sobre os aspectos não médicos do seu contexto de vida, ou seja, uma análise que só pode ser feita pelo próprio indivíduo, ao contrário das tendências iniciais de uso do conceito que direcionavam a avaliação a um profissional de saúde. Quanto à multidimensionalidade refere-se ao reconhecimento de que o construto é composto por diferentes dimensões (SEIDL, 2004).

Um estudo feito pela Organização Mundial da Saúde (OMS) em 1994 (UFRGS, 2010), que teve como objetivo a criação de instrumentos medidores de qualidade de vida, classificou-a com um sentido mais amplo. Observou-se uma multidimensionalidade do conceito, surgindo assim uma estrutura composta por seis domínios: domínio I (físico): dor, desconforto, energia, fadiga, sono e repouso; domínio II (psicológico): sentimentos positivos, autoestima, aparência, sentimentos negativos, etc.; domínio III (nível de independência): mobilidade, atividades da vida cotidiana, uso de medicação e tratamentos, capacidade de trabalho/produção, etc.; domínio IV (relações sociais): relações interpessoais, apoio social, atividade sexual, etc.; domínio V (ambiente): segurança física e proteção, recursos financeiros, lazer, ambiente físico poluição, ruído, trânsito, clima - etc.; domínio VI (aspectos espirituais, crenças pessoais ou religião): espiritualidade, religião e crenças pessoais.

Nesse caso, percebe-se claramente que o ambiente - domínio V, com destaque para lazer, além do ambiente físico propriamente dito - torna-se um elemento relevante para a avaliação da qualidade de vida, principalmente no contexto urbano, no qual a poluição do ar, a sonora e a visual podem influenciar diretamente no bem-estar da população (UFRGS, 2010). No entanto, o tema qualidade ambiental é bastante complexo, bem como seus padrões e seus indicadores. Fatores subjetivos que levam em conta a percepção que o indivíduo tem em relação ao seu ambiente e ao seu próprio modo de vida estão contidos nesses indicadores (SILVA, 2002). Além disso, existem os fatores objetivos: econômicos, sociais, culturais e políticos, que se manifestam distintamente no espaço (SILVA, 2002), possibilitando diversos tipos de interpretações, e tornando difícil de chegar a uma conclusão.

A despeito dessa dificuldade, há, porém, um consenso quando se relaciona qualidade ambiental urbana e vegetação. Considera-se que a qualidade ambiental satisfatória é determinada por composições paisagísticas que privilegie, sobretudo, mas não somente, a vegetação; vista desde um simples gramado às mais diversas espécies arbóreas. Nessas condições, a vegetação constitui componente chave, embora outros componentes também sejam necessários para o alcance de um padrão mínimo de qualidade do ambiente (GOMES; SOARES, 2004).

Sobre essa relação, Richter; Bocker afirmam:

A qualidade ambiental urbana está diretamente ligada ao acesso dos moradores à quantidade, qualidade e distribuição de espaços livres de construção que possam permitir um saudável contato com a natureza, propiciando também possibilidades de socialização e expressão cultural; portanto, uma combinação entre conservação da natureza, conservação da flora e da fauna, conservação do solo, funções climáticas e as necessidades da população em relação à recreação e relaxamento em contato com a natureza. Nesse caso, é essencial a implantação de espaços livres urbanos que possam satisfazer os diversos interesses humanos das mais variadas formas (RICHTER; BÖCKER, 1998). 
A manutenção das áreas verdes urbanas sempre foi justificada pelo seu potencial em propiciar qualidade ambiental à população. Ela interfere diretamente na qualidade de vida dos seres por meio das funções sociais, ecológicas, estéticas e educativas que elas exercem para amenização das consequências negativas da urbanização (CAPORUSSO; MATIAS, 2008). O impacto do espaço verde sobre a saúde é cada vez mais reconhecido.

Para Milano (1990, apud CAPORUSSO; MATIAS, 2008) a principal função do sistema de áreas verdes urbanas não deve ser apenas a criação de refúgios para que as pessoas possam "escapar" da cidade. Além disso, estas áreas devem possibilitar à população momentos de lazer e recreação em contato com a natureza, respeitando sua vivência urbana e contato com outras pessoas.

A oferta de espaços verdes seguros, limpos e confortáveis tem impactos na saúde, medidos de forma direta através do estado de saúde auto avaliado e, de forma indireta, através da melhoria da qualidade ambiental. Dado o crescente consenso que o ambiente é a chave em promover expansão da energia, garantir oportunidades para o aumento da atividade física é um meio promissor de combate ao comportamento sedentário associado com uma variedade de doenças crônicas.

Alguns planejamentos urbanos desconsideram o papel multifuncional desses espaços verdes e muitas decisões são baseadas apenas em estética e custo. O que falta em muitos casos é uma visão abrangente do papel dos parques urbanos e a compreensão de que eles formam uma estrutura que pode resultar em qualidade de vida e constituir uma chave na estratégia de saúde proposta por autoridades.

\section{DETERMINANTES FÍSICOS PARA O USO ATIVO DE PARQUES URBANOS}

Os fatores utilizados para medir o uso dos parques variam em termos de período, tempo de atividade, tipos de parques estudados, entre outros. Isso produz estimativas igualmente variadas de prevalência de seu uso. Mensurar o nível de atividade física realizada nos parques e relevância dos mesmos na estratégia de promoção de saúde através de uma população mais ativa torna-se um desafio multidisciplinar.

Nos Estados Unidos e na Austrália as atividades passivas realizadas nos parques superam a prática de atividade física regular (GILES-CORTI et al., 2005). Na Austrália, por exemplo, a prevalência do uso adulto de parques para a atividade física nas duas semanas anteriores ao estudo variou de 13,0\% a 17,3\% do total de frequência do parque (GILES-CORTI et al., 2005). Já no Brasil, um estudo realizado por Reis (2001) na cidade de Curitiba constatou que a maioria da população presente no Jardim Botânico encontra-se fisicamente ativa, e ainda que $61,85 \%$ desses indivíduos atingem as recomendações para a prática de atividade física. À proporção disso, Brownson (2000, apud REIS, 2001) constatou que em 50 estados americanos somente $38 \%$ da população analisada atingiu as mesmas recomendações.

Inúmeros fatores observáveis podem influenciar o uso dos parques urbanos. Uma revisão da literatura por Broomhall (2005, apud GILES-CORTI et al., 2005) incluiu a esses: as características do próprio parque qualidade e quantidade do espaço disponível; o acesso a instalações locais - quadras e centros de lazer; a relação entre atributos do parque e necessidades dos usuários locais; a manutenção do parque e ainda os fatores de segurança percebida.

Em sua proposta conceitual, Bedimo-Rung et al. (2005) propõem um modelo para guiar pensamentos e sugerir hipóteses, e define alguns atributos dos parques que podem ser utilizados como padrão para futuras pesquisas. Nesta classificação, a discussão centra-se sobre as características ambientais do parque que poderiam estar relacionados à atividade física, sendo essas as características do ambiente, condições dos parques, facilidade ou dificuldade de acesso, estética do local, segurança percebida e políticas voltadas a esse objetivo. De outra maneira, Tinsley et al. (2002) atribuíram o uso dos parques a fatores como proximidade, acessibilidade (não existência de rodovias), aspectos estéticos como a presença de árvores, água (lago) e existência de pássaros, manutenção do parque, tamanho e a disponibilidade de facilidades como calçadas ou percursos para pedestres (caminhada).

Em meio à diversidade de variáveis, existe certo consenso encontrado em um número significativo de pesquisas ao se tratar da relação positiva entre o fácil acesso e/ou proximidade a parques urbanos e o aumento do nível de atividade física nesses locais (REIS, 2001; GILES-CORTI et al., 2005; COHEN et al., 2007; KACZYNSKI AND HENDERSON, 2007; KACZYNSKI et al., 2008; SUGIYAMA et al., 2010; COOMBES, 2010; VEITCH et al., 2013). Em estudo recente realizado nos Estados Unidos, participantes relataram-se propensos a visitar parques locais desde que não seja necessário atravessar ou viajar em estrada de tráfego intenso (KACZYNSKI, 2014, apud VEITCH 2014). 
A despeito disso, ao explorar a disponibilidade de acesso a parques urbanos e sua relação com a prática de atividades físicas, muitos autores não encontraram relação alguma em Norwich, na Inglaterra (HILLSDON, 2006), nos parques da Georgia e Missouri nos Estados Unidos (HOEHNER et al., 2005), em estudo nacional realizado na Nova Zelândia (WITTEN et al., 2008) e também na Escócia (ORD et al., 2013). Esses autores partem da premissa que os parques urbanos são influentes na saúde das populações estudadas, mas desconsideram que a prática de atividade física seja o mecanismo que explique essa associação. Esses resultados adversos dificultam a elucidação da temática em questão.

A estética e características do parque também têm sido positivamente associadas à visitação do parque para a manutenção da vida ativa (GILES-CORTI et al., 2005; REIS et al., 2009). Ao revisar 18 estudos, Owen et al. (2004) concluíram que fatores estéticos e a presença colinas estavam diretamente associados a atividades físicas auto percebidas, apesar de nenhum desses estudos reportar qual o tipo de atividade física que ocorreu nesses locais (COHEN et al., 2007). Na cidade de Curitiba, a beleza geográfica do parque e as pistas de caminhada/corrida foram atribuídas como incentivadoras da prática de atividade física por mais de 94\% dos entrevistados (REIS, 2001).

O tamanho dos parques e a proximidade a parques "formais" foram considerados por Coombes et al. (2010) características essenciais na busca de níveis de atividade física e diminuição do sobrepeso. No que concerne a isso, Cohen et al. (2007) concluíram que recursos, mais do que o tamanho do parque, atraem pessoas para a prática de atividades físicas, incluindo acessibilidade, disponibilidade e qualidade das facilidades. Kaczynski et al. (2008), por sua vez, classificam a presença de trilhas - pavimentadas, não pavimentadas e arborizadas - como os determinantes da prática em questão, e desconsidera a influência da estética e segurança do parque nos 33 parques analisados no Canadá.

No Brasil, um estudo feito em Florianópolis por Collet et al. (2008) observaram-se que os equipamentos disponíveis no parque não se apresentaram como fatores relevantes, contrariando os achados de Cohen et al. (2007). A mesma escala de auto percepção do ambiente utilizada por Collet et al. (2008) foi posteriormente utilizada por Silva et al. (2009) em Curitiba. Curiosamente, o indicador "equipamentos disponíveis" se apresentou como fator fortemente estimulador para a prática de atividades físicas. Observouse que no segundo estudo, a população que reportou esse fator como estimulante advinha de famílias com baixo nível sócio econômico. Isso pode indicar que quanto mais baixa a renda do indivíduo, maior a tendência em perceber esse fator como estimulador. Uma possível explicação seria que pessoas de baixa renda têm poucas oportunidades de praticar atividades físicas em locais particulares (clubes e academias) e valorizam ambientes públicos com equipamentos disponíveis gratuitamente (SCOTT; JACKSON, 1996; BEDIMO-RUNG et al., 2005; GORDON-LARSEN et al., 2006; POWELL et al., 2006 apud SILVA; PETROSKI; REIS, 2009).

Quanto ao fator tecnológico e arquitetônico do ambiente no parque, os resultados encontrados em Florianópolis (COLLET et al., 2008) também diferiram quando comparados aos de Curitiba (SILVA et al., 2009). Enquanto Silva et al. (2009) observaram que em Curitiba a pista de caminhada no parque, estacionamento e beleza arquitetônica das estruturas construídas foram fatores estimulantes para a população analisada, Collet et al. (2008) concluiram que no parque de Florianópolis podem ser considerados estímulos positivos somente a estética arquitetônica das estruturas construídas e a pista de caminhada e corrida, sendo considerado indiferente o estacionamento no parque.

Outro estudo realizado em Curitiba associou qualidade ambiental à frequência no uso de parques e praças (CASSOU, 2009). A maioria dos usuários dos parques estudados indicou ser a beleza e presença de estacionamento os fatores mais significativos. Já os frequentadores das praças consideram imprescindíveis a proximidade de casa, estrutura, equipamentos e segurança percebida.

Embora muitos estudos incluam a vegetação presente nos parques no grupo dos "fatores estéticos", outros estudos buscam esclarecer essa relação de maneira mais específica. Em 1999, estudiosos da Filadélfia iniciaram um programa para transformar lotes vagos da cidade em ambientes verdes. Estudos analisaram a saúde e a segurança da população durante os 10 anos da intervenção, e também compararam o resultado final com outros locais vagos onde o programa não havia sido implantado (BRANAS et al., 2011). Os resultados da pesquisa sugeriram que a "verdificação" do lote foi suficiente para reduzir a criminalidade e promover alguns aspectos de saúde. Ademais, alguns pontos que sofreram essa transformação obtiveram significante aumento na prática de exercício físico e também na diminuição no stress (BRANAS et al., 2011). Contudo, o fato de esse aumento só haver alcançado pontos isolados da cidade sugere que normas culturais e/ou sociais podem relacionar-se mais intimamente à prática de exercícios físicos quando comparadas à presença de vegetação urbana. 
Ainda em relação à vegetação, Maas et al. (2008) através de estudo realizado na Nova Zelândia concluem que a quantidade de espaço verde no ambiente de vida é dificilmente relacionada com o nível de atividade física. Para os autores, a quantidade de atividade física realizada em ambientes de vida mais ecológicos não explica a relação entre o espaço verde e saúde.

Isto posta, a presença de espaços verdes públicos nas cidades mostra-se fundamental para a qualidade ambiental e saúde da população, mas somente o fato de existirem não parece garantir os objetivos propostos por essa pesquisa. À essa presença atrelam-se tantos outros fatores supracitados, a fim de que esses ambientes atinjam o nível de atividade física necessário para obtenção de ganhos em saúde.

\section{RESULTADOS}

Foram aplicados 200 questionários utilizando-se de métodos de amostragem probabilísticos á frequentadores do Parque do Povo maiores de 18 anos durante o primeiro semestre de 2015, em dias da semana e horários variados para representar de forma mais abrangente a população estudada.

A Tabela 1 apresenta a caracterização de algumas variáveis sociais e demográficas da população do estudo. Dos 200 frequentadores entrevistados, a maioria eram mulheres (52\%), com a idade entre 26 e 35 anos (33\%), solteiros e casados (47\% e 43\%), que completaram o nível superior (56\%).

Quando questionados em relação aos motivos que os levam à prática de $\mathrm{AF}$, os motivos relacionados a manter e melhorar a saúde tiveram a média mais alta da escala Likert apresentada, seguidos dos motivos relacionados a condição física, psicológicos e aqueles relacionados com o corpo. Os motivos interpessoais obtiveram a menor média nessa questão (Figura 1).

$\mathrm{O}$ ambiente verde obteve a maior média na questão que levantou os motivos que levavam aqueles usuários até o Parque do Povo para a prática de AF. A quantidade de espaço que o parque oferece e o fácil acesso deste também tiveram média acima de 4 em uma escala de 0 a 5 (Figura 2). A menor média nessa questão ficou para o item "ver pessoas e ser visto", que alcançou a média 2,9.

Tabela 1: Apresentação dos resultados da aplicação de questionários.

\begin{tabular}{|ccc|}
\hline Variável & $\mathrm{n}$ & $(\%)$ \\
\hline Sexo & & \\
Homens & 91 & $48 \%$ \\
Mulheres & 109 & $52 \%$ \\
Idade & & \\
18 a 25 & 40 & $20 \%$ \\
26 a 35 & 66 & $33 \%$ \\
36 a 45 & 54 & $27 \%$ \\
46 a 55 & 26 & $13 \%$ \\
56 ou mais & 14 & $7 \%$ \\
Estado Civil & & \\
Solteiro & 94 & $47 \%$ \\
Casado & 86 & $43 \%$ \\
Divorciado & 12 & $6 \%$ \\
Viúvo & 8 & $4 \%$ \\
Escolaridade & & \\
Ensino Superior & 112 & $56 \%$ \\
Ensino Médio & 76 & $38 \%$ \\
Ensino Fundamental & 10 & $5 \%$ \\
Ensino Fundamental Incompleto & 2 & $1 \%$ \\
\hline
\end{tabular}

Fonte: Autores 


\section{Por que você pratica atividades físicas?}

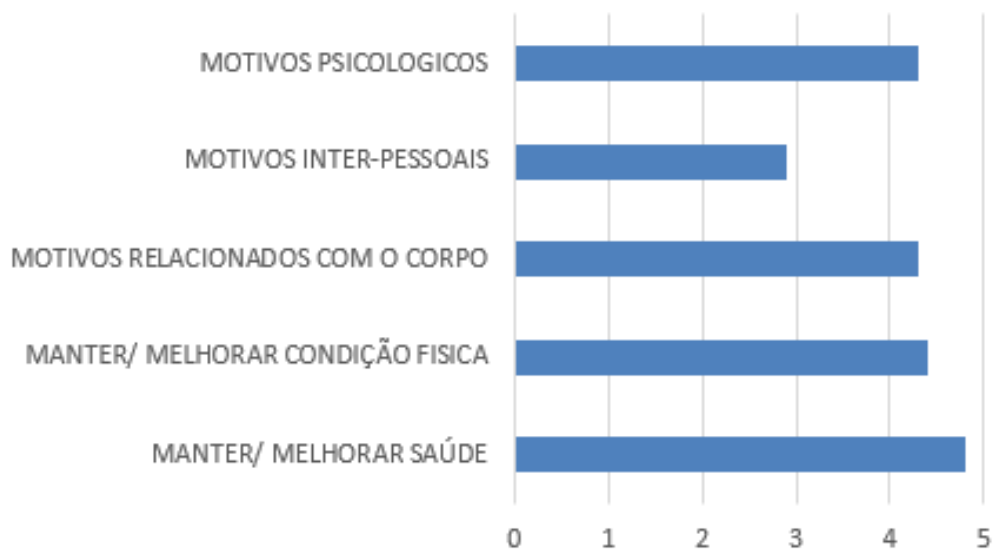

Figura 1. Motivos que levam a prática de atividade física. Fonte: Autores

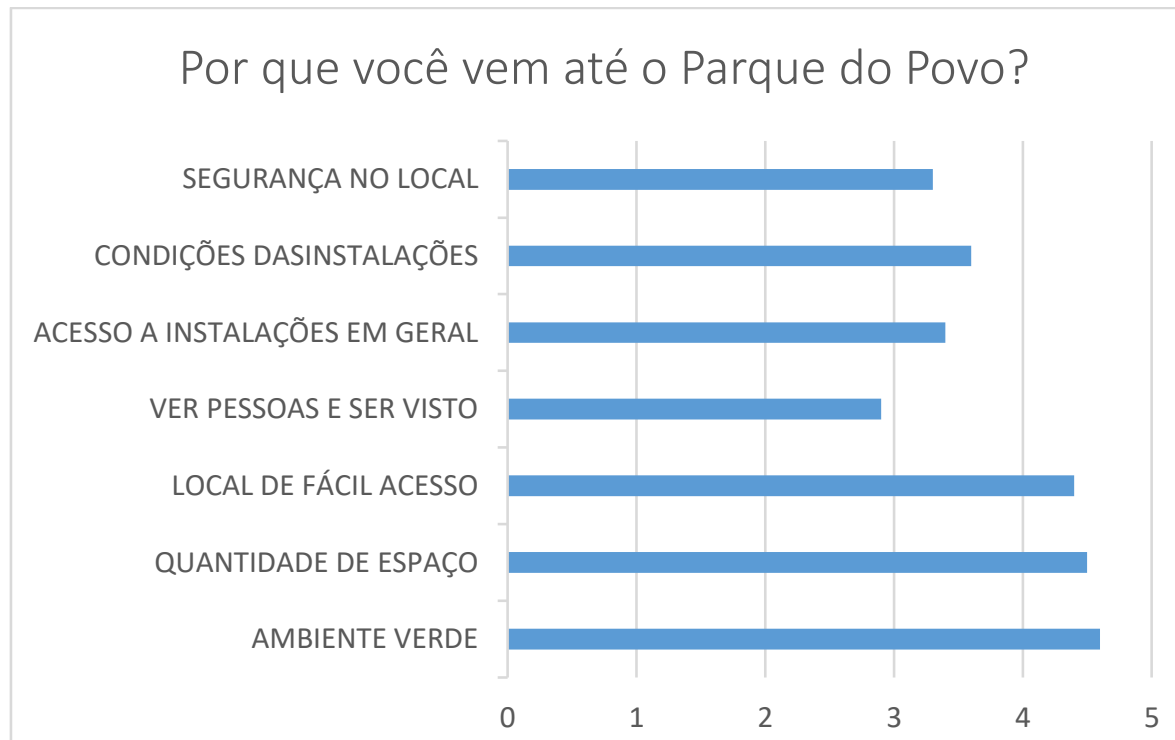

Figura 2. Motivos que levam os usuários até o Parque do Povo para a prática de atividades físicas.

Fonte: Autores

A maioria dos frequentadores do parque relatou não ter tido orientação profissional para a realização da $\mathrm{AF}(67 \%)$, sendo aqueles que procuraram orientação médica ou de profissional de educação física apenas 17\% e 16\% respectivamente (Figura 3). Apenas 5\% dos entrevistados relataram não terem sentido melhora na saúde desde que começou a frequentar o parque para a prática de AF. Em relação ao tempo de deslocamento até o parque, $65 \%$ dos usuários levaram 10 minutos ou menos para chegar até o parque. Apenas 3\% relatou precisar de mais de 30 minutos para a chegada até o local (Figura 4).

O bairro em que os entrevistados residem foram listados, e através disso pode-se observar o raio de abrangência que o parque exerce na cidade (Figura 5). Mais da metade dos frequentadores alegaram permanecer no local por mais de uma hora e menos de uma hora e meia (54\%). Apenas $5 \%$ deles disseram que permanecem por mais de duas horas 


\section{Procurou algum profissional de saúde para realizar essa prática?}

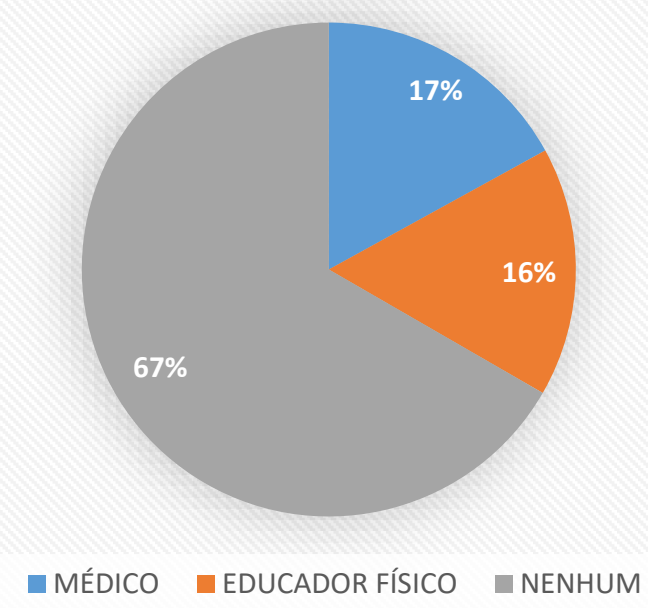

Figura 3. Orientação profissional dos usuários do Parque do Povo para a prática de atividades físicas. Fonte: Trabalho de campo (2015)

\section{Tempo de deslocamento até o parque}

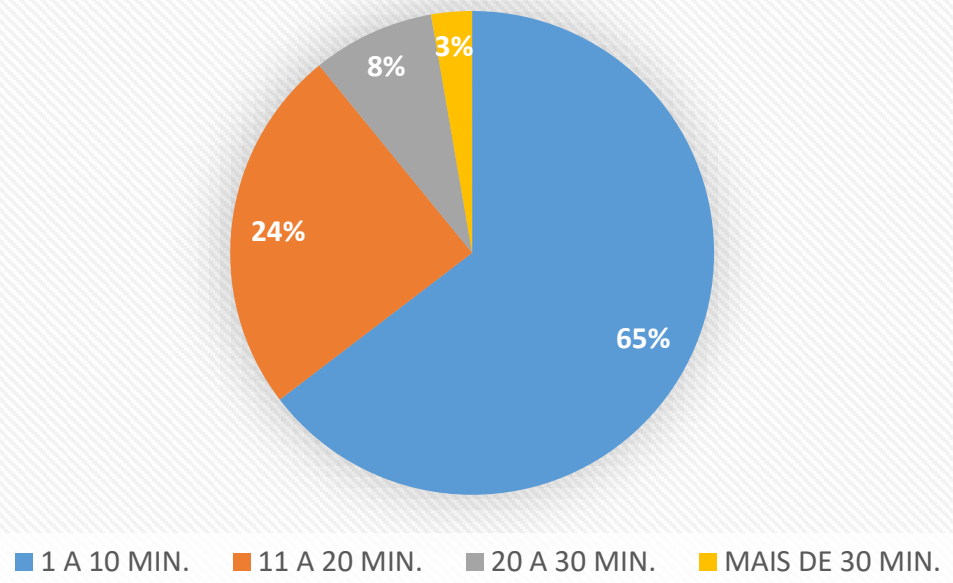

Figura 4. Tempo de deslocamento até o Parque do Povo. Fonte: Trabalho de campo (2015) 


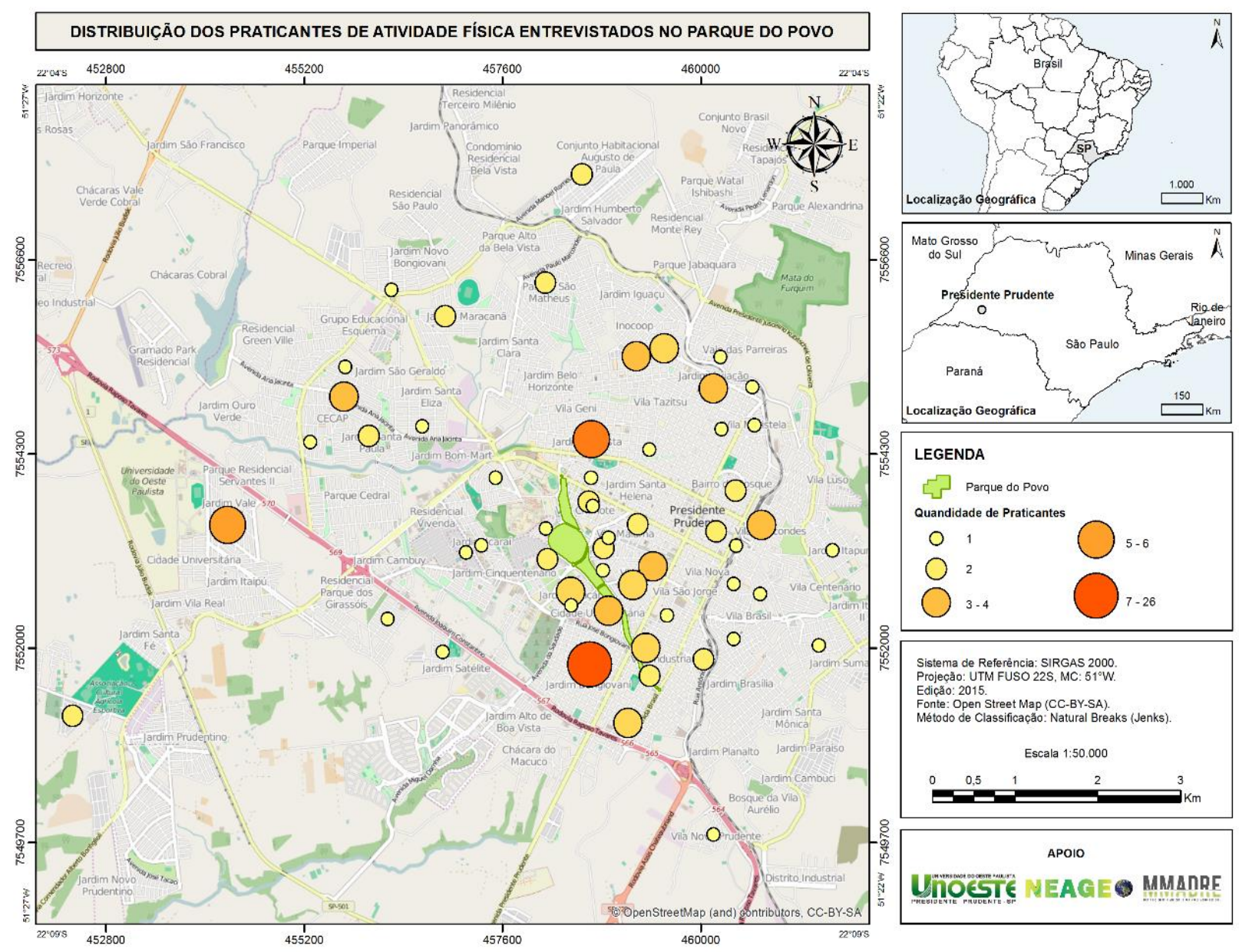

Figura 5: Raio de abrangência do Parque do Povo em relação aos bairros da cidade.

Fonte: Trabalho de campo (2015)

\section{CONSIDERAÇÕES FINAIS}

Os questionários aplicados trouxeram resultados diferentes quanto ao gênero: cerca de metade das respondentes foram mulheres. Observa-se que alguns estudos realizados no Brasil admitiram os mesmos resultados (REIS, 2001; CASSOU, 2009), e também utilizaram instrumentos de autor-relato como meios metodológicos. Incute-se a isso o fato de as mulheres parecerem ser mais susceptíveis a responder questionários, além de estarem menos envolvidos em atividades vigorosas, fatores que colaboram para o aumento da porcentagem do gênero quando esse instrumento é utilizado.

$\mathrm{O}$ fato de os homens estarem mais presentes no parque, se engajarem em mais atividades vigorosas do que as mulheres e ocuparem em número desproporcionalmente maior a área com mais instalações desportivas, pode ser parcialmente explicado através do tipo de atividade escolhida. Evidências mostram que no Brasil, os homens preferem jogar futebol e as mulheres preferem a caminhada como principal AF de lazer (Malta et al., 2009, MINISTÉRIO DO ESPORTE, 2015). Atividades como uma partida de futebol pode envolver vários homens ao mesmo tempo, no mesmo local, o que elevaria o número de indivíduos observados em um dado momento e refletiria nessa discrepância.

O baixo número de crianças observado no estudo (7\%) chama a atenção, já que o parque oferece vários playgrounds, bem como áreas de uso comum que poderiam ser desfrutadas por essa faixa etária. Além disso, o público infantil aumenta aos finais de semana, o que sugere um acordo com os achados de Hino et al. (2010), que constatou que as praças recebem mais crianças que os parques, e que isso se deve ao fator proximidade, já que as praças estariam mais próximas às residências do que os grandes parques. Esse fator acaba por influenciar diretamente na disponibilidade diária dos adultos em acompanhar as crianças para atividades ao ar livre, disponibilidade essa que aumenta aos finais de semana e pode traduzir esse resultado.

A distribuição da população acima de 60 anos também trouxe dados importantes: o período da manhã foi o favorito e sua frequência diminuiu aos finais de semana. Esses achados podem estar atribuídos a peculiaridades próprias da faixa etária, que é adepta a rotinas e vida diurna. Outro fator interessante é que 
uma das áreas menos frequentadas pela população em geral foi preferida por essa faixa etária. Nota-se que essa área possui pistas de caminhada em todo seu interior e arredor, e que, de acordo com Diagnóstico Nacional do Esporte, 80\% dos idosos brasileiros praticaram apenas a caminhada como AF em 2013 (MINISTÉRIO DO ESPORTE, 2015). Esse fator vai de encontro aos estudos de Cohen et al. (2007), que também observaram que o parque com um maior número de pistas de caminhada foi o mais frequentado por idosos. Os autores também sugerem que eles precisam de programas e incentivos para ser atraídos aos parques, e nota-se que as "academias da terceira idade" instaladas ao longo do Parque do Povo destacavamse pela presença dos mesmos ao longo das observações.

O público adolescente representou $21 \%$ da população observada, o que mostra certa importância do local para a faixa etária. Os adolescentes homens foram mais vistos que as adolescentes mulheres (23\%x15\%), fato nitidamente visível nas pistas de skate durante as observações, local de grande concentração do público dessa faixa etária. Nota-se também a presença deles no período do meio do dia, muitas vezes caminhando pelo parque ou descansando nos bancos e locais com sombra de árvores. Esse fator pode estar relacionado ao número de escolas na região e aos seus respectivos horários de entrada e saída.

O nível de AF observado variou muito de acordo com o período do dia. As atividades vigorosas prevaleceram no período da noite (entre $17 \mathrm{~h}: 31 \mathrm{~min}$ e $21 \mathrm{~h}$ ), que foi também o período mais frequentado no total $(53 \%)$, e as atividades sedentárias foram mais observadas por volta do meio dia. Nesse "horário de almoço" foi observada também uma acentuada diminuição da frequência e do nível de AF dos usuários, o que pode sugerir que a maior parte dos frequentadores do parque sejam trabalhadores que buscam exercitarse após o horário de serviço, ou até mesmo que o clima mais ameno no fim de tarde é mais atrativo ao público.

A pesquisa se propôs a entender se a qualidade ambiental trazida por espaços verdes urbanos se configuraria um fator indispensável para a prática de AF nas cidades. $\mathrm{O}$ "ambiente verde" foi o fator mais considerado na escolha dos usuários pelo local da pesquisa, liderando os resultados tanto da escala Likert como da questão aberta do questionário, e demonstrando, então, quão importante se configuram esses espaços na percepção e busca por saúde da população que usufrui deles.

Esse trabalho mostrou uma relação positiva entre áreas verdes urbanas e encorajamento para a prática de $\mathrm{AF}$, revelando que o meio ambiente é uma importante chave para atingir grandes metas em saúde, por ser capaz de influenciar um número maior de pessoas por um período de tempo significativamente superior aos programas individuais de saúde. Sabe-se que uma vida fisicamente ativa traz inúmeros benefícios em muitos determinantes de saúde, e que ações voltadas para esse fim devem receber atenção, já que quase metade da população do país pode ser considerada sedentária. A falta de recursos para participar de atividades ou instalações pagas é uma das principais barreiras na busca de atingir os 150 minutos de AF por semana (Hino et al., 2010), recomendados pela Organização Mundial de Saúde.

\section{REFERÊNCIAS}

AAKER, D. A. Administração Estratégica de Mercado. 5. ed. Porto Alegre: Bookman, 2001.

ARAÚJO, D. S. M. S.; ARAÚJO, C. G. S. Aptidão física, saúde e qualidade de vida relacionada à saúde em adultos. Revista Brasileira de Medicina do Esporte, Niterói, v. 6, n. 5, p.194-203, out. 2000.

BEDIMO-RUNG AL, MOWEN AJ, COHEN DA. The significance of parks to physical activity and public health: a conceptual model. Am J Prev Med. 2005; 28:159-168

BEDIMO-RUNG, A. L.; MOWEN, A. J.; COHEN, D. A. The significance of parks to physical activity and public health: a conceptual model. American Journal of Preventive Medicine, New York, v. 28, n. 2, p. 159-168, 2005.

BRANAS CC, CHENEY RA, MACDONALD JM, TAM VW, JACKSON TD, HAVE TRT. A Differencein-Differences Analysis of Health, Safety, and Greening Vacant Urban Space. American Journal of Epidemiology. 2011.

BROOMHALL, M. H. Study of the availability and environmental quality of urban open space used for physical activity. 1996. Dissertação - University of Western Australia, Perth

CAPORUSSO, D. MATIAS, L.F. Áreas Verdes Urbanas: Avaliação e Proposta Conceitual. Simpósio de Pós graduação em Geografia de Estado de São Paulo - SIMPGEO-SP. 17 à 19 de Dezembro de 2008, Rio Claro/SP. 2011. 
CASSOU, A.C.N. Características ambientais, Frequência de utilização e nível de atividade física dos usuários de parques e praças de Curitiba-PR. Curitiba, 2009. 130 p. Dissertação (Mestrado em Educação Física) - Universidade Federal do Paraná.

COHEN DA, MCKENZIE TL, SEHGAL A, WILLIAMSON S, GOLINELLI D, LURIE N. Contribution of public parks to physical activity.AmJ Public Health 2007;97:509 -14.

COLLET C, CHIARADIA BM, REIS RS, NASCIMENTO JV. Fatores determinantes para a realização de atividades físicas em parque urbano de Florianópolis. Rev Bras Ativ Fís Saúde 2008. 13(1):15-23

COOMBES E., JONES A.P., HILLSDON M. The relationship of physical activity and overweight to objectively measured green space accessibility and use. Social Science and Medicine. 2010;70:816-822.

GILES-CORTI, B., BROOMHALl, M., KNUIMAN, M., COLLINS, C., DOUGLAS, K., et al., 2005. Increasing walking: how important is distance to, attractiveness, and size of public open space? American Journal of Preventive Medicine 28, 169-176.

GOMES MAS; SOARES BR. Reflexões sobre qualidade ambiental urbana. Estudos Geográficos, Rio Claro, 2(2): 21-30 ,jul-dez - 2004 (ISSN 1678-698X)

HILLSDON, M, PANTER, J, FOSTER, C et al., (2006). The relationship between access and quality of urban green space with population physical activity. Public health, 120 (12), 1127-1132.

HINO, A. A. F. et al. Using observational methods to evaluate public open spaces and physical activity in Brazil. Journal of Physical Activity \& Health, Champaign, v. 7, supl. 2, p. S146-S154, 2010.

HOEHNER, C.M.; BRENNAN RAMIREZ, L.K.; ELLIOTT, M.B.; HANDY, S.L.; BROWNSON, R.C. Perceived and objective environmental measures and physical activity among urban adults. American Journal of Preventive Medicine. Amsterdam, v. 28, n. 2, suplemento 2, p. 105-116, 2005.

MAAS J, VERHEIJ RA, SPREEUWENBERG P, GROENEWEGEN PP. Physical activity as a possible mechanism behind the relationship between green space and health: a multilevel analysis. BMC Public Health 2008.

MALTA, D. C. et al. Padrão de atividade física em adultos brasileiros: resultados de um inquérito por entrevistas telefônicas, 2006. Epidemiologia e Serviços de Saúde, Brasília, v. 18, n. 1, mar. 2009.

MARKLAND, D.; INGLEDEW, K. The measurement of exercise motives: Factorial validity and invariance across gender of a revised Exercise Motivations Inventory. British Journal of Health Psychology, Leicester, v. 2, p. 361-376, 1997

MINISTÉRIO DO ESPORTE. Desporte Diagnóstico Nacional do Esporte - Caderno I, Junho- 2015 http://www.esporte.gov.br/diesporte/diesporte_grafica.pdf

MCARDLE, WILLIAM D. Fisiologia do exercício: nutrição, energia e desempenho humano / William D. McArdle, Frank I. Katch, Victor L. Katch; traduzido por Giuseppe Taranto. Rio de Janeiro: Guanabara Koogan, 2011. Il.

MOUTÃO, J. Motivação para a prática de exercício físico: estudo dos motivos para a prática de Atividades de fitness em ginásios. Dissertação (Mestrado em Psicologia Do Desporto e Exercício) Instituto Politécnico de Castelo Branco, Escola Superior de Educação, 2005.

KACZYNSKI A, POTWARKA L, SAELENS B. Association of park size, distance, and features with physical activity in neighbourhood parks. Am J Public Health 2008;98:1451- 6.

KACZYNSKI, A.T., HENDERSON, K.A., 2007. Environmental correlates of physical activity: a review of evidence about parks and recreation. Leis. Sci. 29, 315-354

KACZYNSKI AT, KOOHSARI MJ, STANIS SA, BERGSTROM R, SUGIYAMA T: Association of street connectivity and road traffic speed with park usage and park-based physical activity. Am J Health Prom 2014, 28:197-203.

REIS R, HINO A, FLORINDO A, ANEZ C, DOMINGUES M. Association between physical activity in parks and perceived environment: a study with adolescents. J Phys Act Health 2009;6:503-9.

ORD K, MITCHELL R, AND PEARCE J. Is level of neighbourhood green space associated with physical activity in green space? International Journal of Behavioural Nutrition and Physical Activity. 2013. 10: 127 
OWEN N, HUMPEL N, LESLIE E, BAUMAN A, SALLIS JF. Understanding environmental influences on walking: review and research agenda. Am J Prev Med. 2004;27:67-76.

PESQUISA NACIONAL DE SAÚDE: Percepção do estado de saúde, estilos de vida e doenças crônicas : Brasil, grandes regiões e unidades da federação. Rio de Janeiro. IBGE. 2014.

REIS RS. Determinantes ambientais para a realização de atividades físicas nos parques urbanos de Curitiba: uma abordagem sócio-ecológica da percepção dos usuários. [Dissertação de Mestrado]. Florianópolis: Centro de Desportos - Universidade Federal de Santa Catarina; 2001.

RICHTER, M.; BÖCKER, R. Developing an urban landscape management concept through the integration of environmental quality goals and environmental information systems. In: BREUSTE, J.; FELDMANN, H.; UHLMANN, O. (Orgs.) Urban ecology. Berlim: Springer, 1998. 223p

SHEPHARD RJ, Bouchard C. Principal components of fitness: relationship to physical activity and lifestyle. Can J Appl Physiol 1994;19:200-14

SEIDL, ELIANE MARIA FLEURY; ZANNON, CÉLIA MARIA LANA DA COSTA. Qualidade de vida e saúde: aspectos conceituais e metodológicos. Cad. Saúde Pública, Rio de Janeiro, v. 20, n. 2, Apr. 2004.

SILVA, M. L. G. da. Análise da Qualidade Ambiental Urbana da Bacia Hidrográfica da Lagoa da Conceição. Florianópolis, 2002. 111f. Dissertação (Mestrado em Engenharia de Produção) - Programa de Pós-Graduação em Engenharia de Produção, UFSC.

SILVA, D. A. S.; PETROSKI; E. L.; REIS, R. S. Barreiras e facilitadores de atividades físicas em freqüientadores de parques públicos. Motriz, Rio Claro, v.15, n. 2, p. 219-227, abr./jun. 2009.

SUGIYAMA T., FRANCIS J., MIDDLETON N.J., OWEN N., GILES-CORTI B. Associations between recreational walking and attractiveness, size, and proximity of neighborhood open spaces. American Journal of Public Health. 2010;100(9):1752-1757

TINSLEY H., TINSLEY, D., AND CROSKEYS, C. Park usage, social milieu and psychosocial benefits of park use reported by older urban park users from four ethnic groups. Leisure Sci. 2002; 24: 199-218

UNIVERSIDADE FEDERAL DO RIO GRANDE DO SUL. Psiquiatria. Divisão de Saúde Mental. Grupo WHOQOL. 2006.

VEITCH J, BALL K, CRAWFORD D, ABBOTT G, SALMON J. Is park visitation associated with leisuretime and transportation physical activity? Prev Med. 2013 Nov; 57(5):732-4.

VEITCH, JENNY, CARVER, ALISON, HUME, CLARE, CRAWFORD, DAVID, TIMPERIO, ANNA, BALL, KYLIE AND SALMON, JO. 2014, Are independent mobility and territorial range associated with park visitation among youth?, International journal of behavioral nutrition and physical activity, vol. 11, no. 1, Article number 73, pp. 1-12.

WITTEN K, HISCOCK R, PEARCE J, BLAKELY T. Neighbourhood access to public open space and the physical activity of residents: a national study. Preventive Medicine, 2008. 47 (3) $299-303$. 